\title{
ANATOMY OF VENOUS ABNORMALITIES IN MULTIPLE SCLEROSIS PATIENTS
}

\author{
Alessandro Rasman \\ Department of Political and Social Sciences, University of Trieste, Trieste, Italy
}

\author{
LETTER TO THE EDITOR \\ Phlebological Review 2019; 27, 1: 15-16 \\ DOl: https://doi.org/10.5114/pr.2019.93401 \\ Submitted: 5.12 .2019 \\ Accepted: 19.12 .2019

ADDRESS FOR CORRESPONDENCE
Alessandro Rasman
Department of Political and Social Sciences
University of Trieste
1 P. le Europa
34127 Trieste, Italy
e-mail: alessandro.rasman@libero.it

\section{DEAR EDITOR，}

More than ten years have passed since the publication of the study by Zamboni et al. titled "Chronic cerebrospinal venous insufficiency in patients with multiple sclerosis" [1]. According to the authors, clinically defined multiple sclerosis (CD-MS) is strongly associated with chronic cerebrospinal venous insufficiency (CCSVI), characterised by abnormal venous haemodynamics determined by multiple extracranial venous strictures of unknown origin, a scenario that has not been previously described. The location of venous obstructions plays a key role in determining the clinical course of the disease. However, a number of Doppler ultrasonographic studies, which were primarily conducted by neurologists, have questioned the very existence of CCSVI. This includes a large study by Comi et al. titled "Observational case-control study of the prevalence of chronic cerebrospinal venous insufficiency in multiple sclerosis: results from the CoSMo study" funded by the Italian Multiple Sclerosis Society [2]. To resolve the controversy, I believe that it is necessary to examine the pathology - the main essence of medicine.

Post-mortem examination by Baiocchini demonstrated in two MS patients who died from other causes a marked stenosis of the left internal jugular vein (IJV) at the apex of the angle formed by two heads of the sternocleidomastoid muscle, where the IJVs overlie the carotid artery. It was associated with an ectasia and congestion of the intracranial veins [3].

Diaconu et al., in post-mortem examinations in MS patients and non-MS controls in the IJVs and azygous veins, demonstrated a variety of structural abnormalities and also many anatomic variants [4]. Stenosis of the venous wall stenosis was found with a similar frequency in both groups. However, prevalence of intraluminal abnormalities with possible haemodynamic consequences was higher in MS patients, although the number of examined cadavers was small. These results suggest that MRV studies evaluating venous stenoses may be less sensitive than ultrasonography in identifying abnormalities in CCSVI. In addition, CCSVI ultrasound studies should focus on the evaluation of intraluminal abnormalities.

Coen et al. discovered that the walls of IJVs in MS patients had focal thickening, which in the adventitia was characterised by abundant loosely packed fibres of type III collagen [5]. In the adventitia an inverted ratio between type I and type III collagen, with a higher quantity of the latter, has been described. An assessment of immune cells in all three layers of the wall of the IJV did not demonstrate increased infiltration. In the tunica intima a significant derangement and loss of the endothelial cells have been described. Interestingly, scanning electronic microscopy revealed an absence of the endothelium in the defective jugular valves. Finally, in the adventitia layer the presence of calcifications arranged around the vena venarum has been found. Pedriali and Zamboni believe that published evidence clearly shows the presence of abnormalities in the walls of the IJVs in MS patients [6]. Current studies do not clarify the origin of the pathology of the IJV in MS patients. Congenital, infectious, or even post-thrombotic aetiology have been advocated.

Further research on a larger number of cadavers is needed to understand the clinical significance of venous abnormalities in MS.

The author declares no conflict of interest. 


\section{References}

1. Zamboni P, Galeotti R, Menegatti E, et al. Chronic cerebrospinal venous insufficiency in patients with multiple sclerosis. J Neurol Neurosurg Psychiatry 2009; 80: 392-399.

2. Comi G, Battaglia MA, Bertolotto A, et al. Observational case-control study of the prevalence of chronic cerebrospinal venous insufficiency in multiple sclerosis: results from the CoSMo study. Mult Scler 2013; 19: 1508-1517.

3. Baiocchini A, Toscano R, von Lorch W, Del Nonno F. Anatomical stenosis of the internal jugular veins: supportive evidence of chronic cerebrospinal venous insufficiency? J Neurol Neurosurg Psychiatry 2011; 82: 355.

4. Diaconu C, Staugaitis S, McBride J, et al. A pathologic evaluation of chronic cerebrospinal venous insufficiency (CCSVI). Neurology 2012; 78 (1 Suppl): P05-125.

5. Coen M, Menegatti E, Salvi F, et al. Altered collagen expression in jugular veins in multiple sclerosis. Cardiovasc Pathol 2013; 22: 33-38.

6. Pedriali M, Zamboni P. The pathology of the internal jugular vein in multiple sclerosis. J Mult Scler (Foster City) 2015; 2 1000160 